\title{
\begin{tabular}{l|l} 
POLITIQUES \& & Politiques et management public
\end{tabular}
}

\section{Le plafonnement des carrières : le cas des techniciens de l'environnement, entre contrainte réglementaire et manque de reconnaissance}

When careers get stucked: the case of environmental technicians being trapped between the constraints of administrative rules and the lack of recognition

Daniel Marc

\section{OpenEdition}

\section{Journals}

Édition électronique

URL : http://journals.openedition.org/pmp/1426

DOI : $10.4000 / p m p .1426$

ISSN : 2119-4831

\section{Éditeur}

Institut de Management Public (IDPM)

\section{Édition imprimée}

Date de publication : 1 juin 2008

Pagination : 99-110

ISSN : 0758-1726

\section{Référence électronique}

Daniel Marc, «Le plafonnement des carrières : le cas des techniciens de l'environnement, entre

contrainte réglementaire et manque de reconnaissance », Politiques et management public [En ligne], Vol 26/2 | 2008, mis en ligne le 06 mai 2011, consulté le 02 mai 2019. URL : http:// journals.openedition.org/pmp/1426; DOI : 10.4000/pmp.1426 


\title{
LE PLAFONNEMENT DES CARRIERES : LE CAS DES TECHNICIENS DE L'ENVIRONNEMENT, ENTRE CONTRAINTE REGLEMENTAIRE ET MANQUE DE RECONNAISSANCE.
}

\author{
Daniel MARC ${ }^{1}{ }^{2}$
}

\begin{abstract}
Résumé Le plafonnement de carrière est un concept essentiel de la gestion des carrières. L'évolution des structures organisationnelles publiques et la situation démographique des actifs, en plein " papy boom » en font même un enjeu stratégique pour les gestionnaires des ressources humaines. En effet, ce phénomène vient à toucher de part son ampleur l'ensemble des catégories d'emploi et plus seulement les postes de cadres. Une analyse qualitative basée sur des entretiens nous a permis de le vérifier et de proposer des moyens d'actions adaptés à la population étudiée, celle des techniciens de l'environnement.
\end{abstract}

Mots - Clés

Plafonnement de carrière, technicien de l'environnement, motivation, satisfaction au travail, gestion de carrière

\begin{abstract}
The career plateau is an essential concept of the careers management. The organizational evolution of the public sector and the demographic situation of employees, make plateauing a strategic stake for the administrators of the human resources. Indeed, this phenomenon comes to touch from part its scale all the categories of employment and more only the executives' ones. A qualitative analysis based on conversations allowed us to verify it and to propose means of action adapted to the studied population, that of the technicians of the environment.
\end{abstract}

Key words $\quad$ Career plateau; environment technicians; motivation and satisfaction at work; careers management

\footnotetext{
${ }^{1}$ Office National de la Chasse et de la Faune Sauvage, Direction des Etudes et de la Recherche, B.P. 20, Le Perray en Yvelines, 78612 France

${ }^{2}$ Doctorant Université de Paris 1 Panthéon Sorbonne, C.E.R.G.O.R.S., EA 1461, 1, rue de la Sorbonne, Paris, 75231 France daniel.marc@oncfs.gouv.fr
}

Revue POLITITQUES ET MANAGEMENT PUBLIC, Volume 26, $\mathrm{n}^{\circ} 2,2008$

(C) Institut de Management Public - 2008 
Le plafonnement de carrière comme problème potentiel rencontré par une organisation est né véritablement vers la fin des années 70 [théorisé et défini par l'article de Ference (1977) sur la base de travaux préalables démarrés au début des années 60] et l'attention portée à ce phénomène par les chercheurs n'a depuis cessé de croître. La raison de cet intérêt croissant réside essentiellement dans la diminution des possibilités de promotion constatée dans nombre d'organisations (Tremblay et al., 1995). Le phénomène est dû à deux causes principales. La première est l'évolution démographique de la population active qui du fait du baby-boom laissait prévoir un étranglement des carrières. L'autre est la modification organisationnelle des structures et des carrières qui tend à limiter la linéarité des parcours et l'unicité des carrières qui ne sont plus la norme sociale dominante. Cela entraîne une mobilité vécue et/ou souhaitée, interorganisationnelle plus forte alors que le fait de ne pas progresser d'un poste à l'autre est toujours mal perçu par les employés eux-mêmes et par les recruteurs. Les notions de satisfaction, d'épanouissement au travail et de motivation sont très liées à la promotion verticale. Les conséquences au plafonnement doivent a priori être encore plus élevées lorsque des contraintes réglementaires viennent bloquer encore d'avantage les possibilités de promotion. Mais dans quelle proportion, et avec quels outils accompagner ou modifier cette situation? C'est ce qu'il convient de rechercher par la mise en évidence de la réalité de ce problème pour le corps des techniciens de l'environnement. Créé en 2001, c'est l'un des corps de fonctionnaires les plus récents, élaboré pour doter le ministère en charge de l'écologie de ses premiers agents gérés en régie. En effet depuis sa création dans les années 70 , ce ministère n'avait jamais disposé de personnels propres sur le territoire mais seulement d'agents mis à disposition et gérés par d'autres administrations, l'agriculture et l'équipement en particulier. Pour remédier à cela, et à l'opposé de la démarche de réduction des corps, le gouvernement - Mme VOYNET ministre de l'environnement en exercice en particulier - prit la décision de créer deux nouveaux corps de fonctionnaires par titularisation des personnels techniques de catégorie $B$ et $C$ de l'Office National de la Chasse et de la Faune Sauvage (ONCFS), du Conseil Supérieur de la Pêche (devenu l'Office National de l'Eau et des Milieux Aquatiques) et des Parcs Nationaux, sans toutefois prévoir de corps de catégorie A en débouché.

Après avoir présenté un bref état de la littérature sur le plafonnement, la première partie de cette étude portera sur la vérification de l'état de plafonnement des techniciens de l'environnement, sa caractérisation et les conséquences que cela induit. La deuxième sera consacrée aux solutions techniques et managériales envisageables dans des structures publiques pour limiter les effets du plafonnement. 
La notion de plafonnement dans la littérature
La littérature existante sur le plafonnement des carrières permet d'identifier les sources du problème et suggère des mesures pour y remédier. Plus précisément, les effets négatifs et les mesures d'impact des variables correctrices (managériales et organisationnelles) ont été reprises après analyse de quinze études de cas déjà publiées dans la littérature, portant sur des populations significatives et relativement proches de la population étudiée, celle des techniciens de l'environnement. Les résultats obtenus dans ces études de cas sont relativement contrastés. Les études prises en considération sont échelonnées sur la période 1977-2004, ce qui donne une tendance à long terme et ne résulte donc pas seulement de l'examen d'un épiphénomène contextuel.

Que ce soit en considérant seul le plafonnement objectif dans son acceptation classique, mesuré par l'absence constatée de mouvements, (Choy (1998) et Chao (1990)) ou bien en s'intéressant au plafonnement subjectif ou professionnel, (Chang Boon lee (2002)), ces auteurs identifient clairement un impact négatif du plafonnement sur divers paramètres que sont par exemple: la motivation au travail, la fidélité à l'organisation ou inversement la volonté de la quitter, le bien-être (ressenti et médical), l'absentéisme et la confiance.

D'autres en revanche (Veiga (1981) ; Zaremba (1994)) ne perçoivent pas de différences significatives entre employés plafonnés ou non. Enfin, même lorsqu'ils montrent des incidences négatives dues au plafonnement, notamment la baisse de satisfaction, ils ne voient pas pour autant de conséquences significatives sur les attitudes au travail, la performance ou le désir de promotion... En effet, le principal effet démontré est la baisse de satisfaction au travail, ce qui est intéressant si l'on part du postulat qu'un agent satisfait travaille mieux et plus. Mais ce n'est pas systématique ou du moins pas homogène sur un continuum temporel. Cela peut expliquer que certains auteurs axés sur des critères objectifs - turn-over, absentéisme, productivité - ont plus de mal à montrer les effets négatifs du plafonnement. Ces études auront permis toutefois de mettre en lumière les conditions, le terreau propice à l'apparition ou non des effets négatifs liés au plafonnement. Slocum (1985) a montré que selon le type de management, les employés de deux organisations commerciales d'un même secteur pouvaient ressentir les effets du plafonnement de leur carrière de façon diamétralement opposée, avec des résultats inverses à ceux prévus, montrant des plafonnés moins sujets au turn-over et plus satisfaits par exemple.

Ces travaux seront corroborés par Feldman (1988) qui pense que le contexte environnemental au sein de l'organisation peut supprimer ou faire apparaître des conséquences néfastes au phénomène de plafonnement. Tremblay (2004) confirme les résultats de Gerpott et Domsch (1987) qui montrent que certains types de métiers sont plus sujets que d'autres au plafonnement, démontrant que les professions dites à vocation comme les médecins ou les chercheurs sont moins sujettes au phénomène. Lemire (2003) identifie des effets négatifs à la situation de plafonnement (objectif et subjectif), tels qu'admis par la plupart des auteurs mais en démontrant que des pratiques managériales telles que l'accompagnement dans la carrière, le développement des tâches ou la reconnaissance (rôle de mentor, de conseiller) peuvent les limiter. La présence de ces pratiques dans certaines des populations étudiées peut expliquer des résultats non significatifs obtenus par d'autres chercheurs. 
En définitive, la littérature nous invite à retenir le plafonnement comme élément générateur d'effets pervers dont les manifestations varient au sein des organisations au gré de leur culture, des pratiques de gestion qui y ont cours ou des caractéristiques de la population envisagée.

L'importance des effets négatifs liés au plafonnement est toujours d'actualité, notamment du fait que le « papy-boom » n'arrivera à terme que dans 6 à 10 ans. Paradoxalement, il semble que les études ciblant le plafonnement de carrières se soient ralenties ces dernières années au profit de notions connexes telles que le plafond de verre.

\section{Mesure du plafonnement professionnel des techniciens de}

l'environnement
Quelle est la définition concrète et exacte du plafonnement? Cette question a été soulevée par Tremblay, Roger et Toulouse (1995) qui en sont arrivés à la conclusion qu'il n'y a pas de définition universelle. Ils proposent d'ailleurs de prendre en considération l'ensemble des moyens de mesure du plafonnement, qu'ils soient objectifs, subjectifs ou professionnel. Dans la plupart des études menées, le plafonnement de carrière est présenté comme le moment où la probabilité d'obtenir une promotion est quasiment nulle. Cette définition a été proposée par Ference et al. (1977): « Le plafonnement se définit comme le stade d'une carrière où la possibilité d'une promotion hiérarchique est très faible ". Slocum et al. (1987) ont ajouté au manque de promotion celui de mobilité horizontale.

Ce plafonnement objectif se mesure classiquement par le temps passé sur un poste ou un niveau (catégorie) ou par le temps écoulé depuis la dernière promotion. La plupart des auteurs considèrent un employé comme étant plafonné lorsqu'il est depuis une durée donnée sur un même poste. Cette durée varie de 5 (Tremblay et Roger (1995)) à 10 ans (Gerpott et Domsch (1987)), avec une moyenne établie à 7 ans (Choy et Savery (1998)). Ces chiffres correspondent aux durées moyennes passées entre deux postes aux responsabilités différentes sur les populations étudiées. Dans la fonction publique française, les seuls postes pour lesquels une durée est indiquée (détachement, mise à disposition, postes fonctionnels) donnent une fourchette comprise entre 3 et 5 ans. La durée de 7 ans (plus de deux périodes de mise à disposition) semble être pertinente pour lancer une étude au sein de la fonction publique, avec la limite que ces positions concernent souvent des postes de cadres. Le principal défaut de cette définition est un certain déterminisme du plafonnement, essentiellement caractérisé par le nombre d'année dans le poste. II s'ensuit que des facteurs plus subjectifs ne sont guère pris en compte alors que le ressenti des agents peut différer d'une organisation à une autre ou dans le temps. Le plafonnement subjectif est d'ailleurs une notion plus récente, apparue dans les années 90, qui doit permettre précisément de dépasser ces limites. Le plafonnement est ici reconnu dès lors que l'agent perçoit et ressent les effets liés au plafonnement. La durée dans le poste ou l'âge sont considérés, mais comme simples variables correctrices ou explicatives, mais elles ne suffisent pas à rendre compte du sentiment de plafonnement. L'élément essentiel est la perception qu'ont les employés d'être restés trop longtemps sur leur poste, d'être peu motivés par les enjeux liés à leur fonction, de mériter une mobilité géographique... II est à noter que les effets négatifs du plafonnement de carrière sont plus importants lorsque les individus ont le sentiment d'être plafonnés que lorsque qu'ils le sont de façon objective (Ettington 1998, Chao, 1990 et Tremblay, 1995, repris par Lemire, 2003). La 
problématique du plafonnement de carrière se rapproche alors de la théorie du contrat psychologique (Rousseau, 1990). Cette notion permet de mieux analyser les comportements des employés en mettant en avant les liens informels et personnels tissés en dehors de tout cadre conventionnel ou réglementaire strict. La confiance, les espérances déduites de l'atteinte d'objectifs revêtent une importance fondamentale dans l'attitude des agents et bien souvent ce n'est qu'en cas de rupture de ce lien que se font ressentir les effets du plafonnement vécu comme une injustice et non comme une évolution normale. Une approche plus récente, celle de plafonnement professionnel (Chang boon lee, 2002) réunit les plafonnements subjectifs et objectifs. Cette définition prend en compte les éléments objectifs tels que la durée dans le poste occupé, l'âge ou l'évolution de carrière en y ajoutant conjointement des éléments de compréhension plus fins comme l'évolution des missions et des responsabilités confiées et les éléments subjectifs, ressentis par les agents. C'est cette notion extensive que nous nous proposons de retenir car elle évite les limites d'une analyse partielle (en termes de plafonnement soit objectif soit subjectif). La population des techniciens de l'environnement semble a priori plafonnée objectivement, du moins sur un plan réglementaire. Outre ce facteur objectif, la définition retenue permettra de cerner d'autres causes et éventuellement de proposer des solutions tant réglementaires que managériales.

Dans cette perspective, la méthodologie envisagée pour montrer la situation de plafonnement des techniciens de l'environnement est différente de celle utilisée habituellement.

En effet, les études de cas recensées dans la littérature et réalisées sur des populations relativement proches (techniciens, agents publics, cadres intermédiaires, postiers...) et de taille significative ont toutes été établies par des méthodes quantitativistes, tant pour identifier la présence ou l'absence d'effets négatifs liés au plafonnement que pour proposer des solutions opérationnelles. Toutefois, une approche qualitative peut être pertinente d'autant que les auteurs les plus spécialisés sur cette thématique (Tremblay, Ettington ou Lemire) ont montré que le plafonnement subjectif - le ressenti donc - est plus important que les éléments objectifs quant aux effets de la notion étudiée. Aussi, pour savoir si la population des techniciens de l'environnement est plafonnée et pour évaluer les effets concrets de ce phénomène, la méthode choisie sera une triangulation de données à forte dominante qualitative. II s'agira tout d'abord de vérifier la présence du plafonnement structurel par la mesure de la possibilité réelle de promotion de ces agents. Elle sera examinée par les dispositions réglementaires relatives à la gestion des corps de l'environnement, aux conditions d'accès au corps de catégorie $\mathrm{A}$ et aux attributions de promotions depuis la création d'un corps de débouché, celui des Ingénieurs de l'Agriculture et de l'Environnement (Décret 2006-8). Ensuite, il a été procédé à des entretiens dirigés, qui ont concerné une dizaine de personnes a priori à même de connaître et d'évaluer la situation des Techniciens de l'Environnement (TE), pour juger de deux critères importants : la possibilité de promotion des TE et le sentiment de plafonnement éventuel. Sont concernés trois représentants syndicaux, quatre représentants de la ligne hiérarchique de l'Office National de la Chasse et de la Faune Sauvage ${ }^{3}$, et trois personnes chargées de la gestion de ce corps dans les ministères de tutelles (ceux en charge de

\footnotetext{
${ }^{3}$ L'ONCFS est le plus gros employeur de TE avec 362 agents, devant l'ONEMA et les Parcs Nationaux.
} 
l'écologie et de l'agriculture). Le questionnaire utilisé doit permettre de resituer le contexte de la création du corps des TE et de dresser un bilan en terme de gestion des carrières après cinq ans de mise en œuvre (les entretiens ont été réalisés en 2006). Les questions doivent permettre de rappeler le contexte politique et social, avant et après la création de ce corps et de disposer du ressenti, qu'il provienne de l'organisation hiérarchique ou des agents.

De plus, les dossiers personnels des 35 TE affectés dans l'une des directions opérationnelles de l'ONCFS ont été examinés (et notamment via les entretiens d'évaluations) afin d'y rechercher des traces attestant la présence des effets d'un éventuel plafonnement. Les éléments recherchés sont ceux laissant à penser que les TE ont tendance à vouloir quitter l'organisation, perdent leur motivation ou ressentent le besoin de promotion. Enfin, l'âge des TE, seule variable individuelle sociodémographique corrélée aux effets négatifs du plafonnement (Lemire 2003), sera abordé sous deux angles : l'âge moyen de la population et la structuration de la pyramide des âges pour simuler l'évolution future des promotions possibles.

Les conclusions issues de cette analyse confirment le plafonnement de cette population. En effet, les techniciens de l'environnement (TE), ont vu au moment de leur titularisation, leur carrière reconstituée dans leur nouveau grade avec de meilleures perspectives salariales et de mobilité horizontale, liée à leur statut (seule la mise à disposition, rarement utilisée, leur était ouverte en tant que non titulaires). Par contre l'absence de création simultanée de corps de catégorie A de l'environnement, alors même que ces postes, au statut de non titulaires, existent dans les établissements publics, bloquait de fait leur mobilité verticale. L'Administration n'a pas méconnu cet état de fait et a proposé de créer un corps de débouché, celui des ingénieurs de l'agriculture et de l'environnement (IAE). Ce nouveau corps issu de la fusion des corps d'ingénieurs des travaux de l'agriculture (ruraux, des eaux et forêts, et de l'agriculture) est ouvert en débouché au corps des TE (cf. décret du 4 janvier 2006). Mais les modalités d'accès au corps des IAE sont très restrictives pour les agents de l'environnement du fait de la nature des épreuves du concours interne et de l'examen et plus généralement des métiers que cela concerne, qui restent très différents de ceux de l'agriculture. Le décret $2006-8$ et ses arrêtés d'applications prévoient des filières de spécialités mais aucune qui concerne les spécificités des TE (police de l'environnement et de la chasse en particulier, aide à la recherche en biologie...).

Par ailleurs, le corps des IAE est géré exclusivement par le ministère en charge de l'agriculture qui se doit de conserver un débouché à ses propres techniciens alors même que le nombre de postes IAE est, dès sa création, en forte diminution. Le nombre total d'IAE prévu étant inférieur au nombre d'ingénieurs des travaux des corps avant fusion. Rappelons que Bardwick (1986) considère que la lutte contre le plafonnement est essentielle lorsque la tendance dans les organisations est à la baisse des effectifs. Si cela est vrai dans beaucoup de secteurs (du fait de la volonté récurrente de diminuer le nombre de fonctionnaires) le phénomène est particulièrement patent pour les techniciens de l'environnement du fait de la concentration en cours des services affectataires (fusion des Directions Départementales de l'Agriculture et de la Forêt et celles de l'Equipement, diminution par 
regroupement du nombre d'agences territoriales à l'Office National des Forêts, fusion des Directions régionales de l'Environnement et des Directions Régionales de l'Industrie, des Risques et de l'Environnement). Cette concentration se traduit par une diminution des postes d'encadrement, c'est à dire l'aplatissement des structures, et par une limitation du nombre d'organisations potentielles d'accueil. Ensuite les postes de catégorie A au sein des établissements publics de l'environnement (EP) ne sont pas des postes IAE ouverts aux concours et examens professionnels du ministère en charge de l'agriculture (postes de non titulaires). Cela suppose que les promus quittent le service public de l'environnement sans savoir quelle fonction ils seront amenés à avoir.

Dans la pratique, depuis 2003 aucun poste d'IAE n'a été pourvu par un technicien de l'environnement de l'ONCFS et pour l'ensemble du corps (tous EP confondus) un seul poste est attribué par an via la liste d'aptitude. Le bilan des cinq dernières années fait apparaître qu'il n'y a pas eu un seul promu au grade d'IAE parmi les agents en poste à l'ONCFS et qu'ils ne peuvent plus postuler sur les postes de catégorie A propres à l'ONCFS (non titulaires) autrement que par le concours externe après démission... (cf. Décret du 29 décembre 1998).

Outre ce plafonnement structurel (causé par l'organisation), s'ajoutent à la situation des TE des facteurs aggravants. Cardinal et Lamoureux (1990) ont montré qu'en plus des facteurs biologiques (telle l'atteinte de l'âge moyen), le plafonnement est un des trois éléments déterminants au déclenchement de la crise du mitan. Cela est d'autant plus vrai que l'on a une profession où la concurrence est élevée et où l'évolution verticale est la plus appréciée dans l'organisation. Selon leurs travaux, une population masculine à plus de $90 \%$, à profession compétitive où seule la mobilité verticale est synonyme de réussite et dont la moyenne d'âge est de 45 ans est à considérer comme plus sensible au phénomène de plafonnement.

Or, la population totale des 362 TE de l'ONCFS, composée à près de $95 \%$ par des hommes ${ }^{4}$ s'élève à 45 ans ce qui correspond à la tranche d'âge la plus sujette aux facteurs aggravants. Par exemple, c'est l'âge de la crise du mitan qui est une remise en cause conduisant à évaluer son parcours antérieur, tant professionnel que personnel visant à faire la balance des ambitions atteintes ou en passe de l'être et des déceptions ou regrets. Ceci à l'âge où s'observe classiquement une certaine stabilisation (Cardinal et Lamoureux 1990).

Il est d'ores et déjà légitime de penser que le plafonnement objectif observé sera ressenti, ce que confirme l'analyse des questionnaires. L'intégralité des interrogés affirme que les TE sont plafonnés. Cette affirmation est apparue directement, sans sollicitations. Toutes les personnes interrogées ont abordé le manque d'une catégorie d'encadrement dans les corps de l'environnement capable de donner un déroulement de carrière aux techniciens. Le corps des IAE n'est pas méconnu, mais jugé inadapté et ne concernant que les agents du ministère de l'agriculture et de l'ONF $(80 \%$ des répondants). Même si la création de ce corps laisse la possibilité théorique d'accéder à la catégorie $\mathrm{A}$, elle est en pratique trop limitée et peu accessible à des agents chargés essentiellement de missions de police de l'environnement. Les conséquences à ce plafonnement apparaissant dans les réponses sont avant tout la démotivation. Elle serait provoquée par un blocage des gains salariaux et au besoin frustré d'accomplissement ou de

\footnotetext{
${ }^{4}$ (Source : ONCFS - Bilan social 2006)
} 
reconnaissance des agents. Les représentants des ministères de tutelle ajoutent la perte d'efficacité due au manque d'émulation entre les individus les plus brillants. Enfin, selon leurs dires, la non reconnaissance des compétences propres à ces agents (en matière de police de l'environnement notamment), conduirait à un appauvrissement de la culture de l'organisation et au turn-over des individus les plus fraîchement recrutés. Pour confirmer ces premiers résultats, un échantillon de 35 TE issu de la même direction, représentant $10 \%$ de la population totale a été étudiée. Ce faible pourcentage et les nombreux biais possibles (management propre à cette Direction, conditions de rémunération différentes, âge moyen plus élevé...) ne permettraient pas à l'échantillon, seul, d'avoir une validité externe suffisante pour établir la présence ou l'absence de plafonnement. Toutefois, recoupés avec ceux des autres méthodes, les résultats peuvent donner des indices objectifs, chiffrés intéressants s'ils corroborent ou contredisent très fortement les hypothèses émises quant au plafonnement des TE. Pour vérifier cet état, ce sont les conséquences du phénomène de plafonnement qui sont recherchées. II ressort que $40 \%$ des agents montrent une baisse de la motivation (déclarée $26 \%$ ou perçue $14 \%$ ) ; $38 \%$ ont demandé une mobilité ou un changement de spécialité ; $29 \%$ font valoir leur niveau d'ingénieur et leur volonté d'y être promus (formation, demande expresse, renseignements). Au final, seul un tiers de la population ne présente aucun des signes caractérisant une population plafonnée alors que $43 \%$ répondent positivement à plusieurs des 9 critères retenus (entre 2 et 7 !). La démotivation est parfois expliquée par les agents dans les entretiens individuels. Les causes principales sont le plafonnement de contenu (plus de challenge à relever, besoin de nouveaux centres d'intérêts) et la recherche inaboutie de promotion.

Les résultats obtenus sont tous convergents et indiquent que la population des techniciens de l'environnement est plafonnée objectivement, et le fait qu'ils en soient conscients (résultats des entretiens et de l'analyse du panel de TE), induit qu'ils sont aussi plafonnés subjectivement. Nous observons que la mobilité horizontale ne semble pas permettre de compenser en totalité le plafonnement vertical puisque les effets négatifs que la littérature attribue au plafonnement sont perçus par les agents et par la ligne hiérarchique alors que le mobilité horizontale n'a jamais été aussi réelle. En effet, le statut de fonctionnaire obtenu par ces agents lors de la création du corps de TE leur permet, contrairement à leur ancienne condition de contractuel, de postuler aisément à la plupart des emplois publics de techniciens.

\section{Solutions managériales et} réglementaires
Après avoir démontré que cette population pâtit effectivement des effets du plafonnement de leur carrière, il convient effectivement d'identifier quelles pratiques ou quels outils, validés de façon théorique et empirique, permettraient d'améliorer cette situation.

Le plafonnement des TE découle essentiellement de l'absence de réels débouchés dans un corps de A propre à la sphère de l'Environnement. Les solutions envisageables à cette situation semblent être d'ordre technique, réglementaire, avant d'être managérial. C'est en tout cas l'avis des personnes chargées de la gestion de ce corps (CGAAER; IGE ; services $\mathrm{RH}$ des EP). Cette vision des objectifs à atteindre semble limitée par le fait 
que les mesures réglementaires doivent pour porter leurs effets être appropriées, comprises et utilisées par les personnes concernées. Cela passe par des pratiques managériales (cf. infra).

Les personnes interrogées, ne proposent pourtant qu'une action d'ordre réglementaire :

La création d'une filière environnement au corps des IAE qui permettent d'intégrer suffisamment les compétences recherchées et appréciées par les services des EP, dans les processus d'évaluation des concours et examens internes.

La transformation des postes d'ingénieurs des travaux des EP en IAE ce qui augmenterait les capacités de promotion et permettrait aux promus de rester dans le service public de l'environnement. En corollaire, il serait nécessaire que le corps soit géré de façon interministérielle comme les attachés des instituts régionaux d'administration ou les administrateurs civils. Ces solutions permettraient effectivement de supprimer le plafonnement organisationnel qui bloque objectivement l'ensemble des carrières des TE, mais pour nécessaires qu'elles soient, elles en demeureraient insuffisantes, pour deux raisons. Tout d'abord, ces mesures n'influent pas sur des facteurs essentiels comme l'âge moyen de la population ou l'évolution du nombre de promotions. Ensuite, il faut aider les agents à valoriser leur parcours pour leur permettre d'évoluer par cette voie nouvelle. Un accompagnement au changement est nécessaire. Le sentiment de plafonnement, lui, ne pourra être éliminé avant qu'un nombre suffisant de promotions effectives permette de rendre un espoir de déroulement de carrière. Or, nous l'avons vu, c'est précisément le plafonnement subjectif qui engendre le plus d'effets négatifs. II faut également ajouter que l'ensemble des facteurs aggravants cités par les différents auteurs considérés affecte la population des techniciens de l'environnement. II s'agit de l'âge moyen de la population qui est élevé (45 ans) avec 39\% des TE qui ont dépassé les 50 ans ; de la durée moyenne des postes supérieure à 7 ans; la forte proportion de techniciens au stade de fin de carrière (plus de $16 \%$ au grade de Chef de technicien, dernier grade du corps) et une concurrence que l'on peut considérer comme très élevée dans le cadre d'éventuels concours internes liée au fait que les recrutements concernent essentiellement des surdiplômés depuis 2003 (deux tiers des recrutés ayant un diplôme supérieur ou égal à la licence selon l'IFORE - Institut de FORmation de l'Environnement pour un niveau Baccalauréat requis) ce qui est par ailleurs un facteur d'apparition des effets négatifs liés aux plafonnement selon Lemire (2003).

Parmi les solutions managériales qui paraissent efficaces, l'accompagnement dans la carrière figure en bonne place. Cet outil est d'ailleurs déjà utilisé pour les populations les plus exposées, celles des cadres supérieurs et dirigeants. Ainsi, la gestion du plafonnement des cadres issus des grands corps d'ingénieurs ou Enarques est déjà bien connue et réellement prise en considération [Bureaux dédiés à leur gestion dans chaque ministère affectataires par exemple].

Si des indices à ces situations de plafonnement font de brèves apparitions dans les débats publics au sujet des non cadres ou des cadres intermédiaires $^{5}$, il est clair que la population des techniciens de

\footnotetext{
${ }^{5}$ (Syndicat national des agents de la C.C.R.F. - CGT; DGCCRF 2008 : et maintenant ? 01 mars 2005. Tract sur le problème du plafonnement à la DGCCRF ; Syndicat National de l'Environnement - FSU ; Flash info du 21 octobre 2005 : Projet de statut des Ingénieurs de l'agriculture et de l'environnement. ; Le Parisien ;
} 
l'environnement n'en est plus à laisser des indices de son plafonnement mais qu'il est à présent évident. Elle doit être également estimée comme à risque et bénéficier à ce titre de telles mesures de gestion des carrières.

L'amélioration des pratiques de gestion des ressources humaines pour limiter les effets pervers que sont la démotivation et le turn-over sont nécessaires, mais lesquelles sont efficaces et adaptables aux fonctionnaires ? La littérature visée en référence nous permet de répondre à ces questions. Parmi les différentes études de cas, près de 20 solutions managériales ont été envisagées pour lutter contre le plafonnement (conseil, mentoring, licenciement, mutation, gestion par projets, intéressement, participation aux décisions, rémunération, développement professionnel, développement personnel, formation, mobilité interne, planification de carrière, évaluation des aptitudes, soutien à la carrière, planification de la relève, outplacement).

Si certaines mesures, comme le licenciement des individus plafonnés inefficaces, proposées par Applebaum et al. 1994 ou encore l'intéressement aux résultats commerciaux ne semblent pas pouvoir concerner les techniciens de l'environnement, d'autres concernant la gestion des carrières pourraient être très efficaces. Lemire 2003 a confirmé par une étude de cas sur la fonction publique Québécoise l'impact positif du soutien et de la planification des carrières, de l'évaluation des aptitudes, de l'encouragement à la mobilité interne et du développement professionnel (participation aux décisions, projets de long terme, planification de la relève) pour limiter les effets du plafonnement. Dans l'étude de Choy et Savery 1998, qui a également considéré une population d'agents publics, on retrouve le soutien (accompagnement, counseling...), l'enrichissement des postes (compétences, projets...), et l'utilisation des compétences (expérience) pour faire d'eux des mentors. II est intéressant de noter que dans une étude de cas sur les services postaux du Royaume-Uni (Zaremba 1994) ce qui est jugé utile effectivement par les sondés sont les mesures de formation qui incluent des diplômes universitaires pour certains, le style de management (intérêt, de l'encouragement et du soutien) et la possibilité de mobilité ou de développement. Si l'on ajoute les résultats des travaux d'Ettington et Tremblay il devient indéniable que ces solutions sont réellement opérationnelles sur les différents panels étudiés et font consensus.

Elles passent par des préalables que sont la Gestion Prévisionnelle des Emplois et des Compétences (GPEC) et l'amélioration des systèmes d'évaluation de la performance et la rétroaction au sujet des résultats (Feldman et Weitz 1988). Ces outils "préalables » sont mis en place au sein de l'ONCFS, rendant possible les solutions managériales envisagées ci-dessus.

Pour la population des techniciens de l'environnement, la solution passe donc par l'application d'une réforme statutaire du corps des IAE à laquelle il faudra obligatoirement ajouter la mise en œuvre de pratiques managériales nécessaires.

Ces pratiques sont, le soutien, la formation et l'information pour le développement des carrières, l'enrichissement des postes et le développement (valorisation) de la mobilité pour pallier les effets du cloisonnement des TE mis en évidence dans cette étude. 
Le cas du corps des techniciens de l'environnement montre qu'il est important de mieux identifier les populations en situation de plafonnement. Les études à venir devraient permettre de caractériser les populations particulièrement exposées aux effets du plafonnement afin qu'un effort ciblé puisse être porté à leur gestion. L'élaboration d'un outil de diagnostic laissant une grande place au ressenti - le plafonnement subjectif étant le plus nocif - paraît être un préalable nécessaire. Ce que nous pouvons retenir de ce qui précède, en guise de canevas de base à un tel outil peut se résumer par une liste d'éléments mesurables comme l'identification des grades ou corps sans débouchés ou a débouchés très limités, la remontée de revendications syndicales à ce propos et le turn-over. Ces éléments factuels gagneraient à bénéficier de l'analyse des entretiens d'évaluations. Ce dernier outil permet en effet de disposer du sentiment des agents quant à leurs perspectives de carrière et leur désir de formation, indispensables pour identifier les individus ressentant les effets du plafonnement. 


\section{BIBLIOGRAPHIE}

BARDWICK, J.; 1986; The Plateauing trap; New-York: Amacom ROUSSEAU, D.M. ; 1990 ; New hire perceptions of their own and their employers obligations: a study of psychological contracts ; journal of organizational behaviour; vol:11; 389-400

CARDINAL, L. ; LAMOUREUX, C. ; 1990 ; La crise de la mi-carrière : état de la question; Carriérologie, vol.4, $n^{\circ} 2,8 p$.

CHANG BOON LEE, P.; 2002; Going beyond the career plateau. Using professional plateau to account for work outcomes ; Journal of management development, vol. 22, $\mathrm{n}^{\circ} 6,538-551$.

CHAO, G.T.; 1990; Exploration of the conceptualization and measurement of career plateau: a comparative analysis; Journal of management, vol.16, $\mathrm{n}^{\circ} 1,181-193$.

CHOY, R.M.; SAVERY, L.K.; 1998 ; Employee plateauing: some workplace attitudes; Journal of management developpement, vol.17, $n^{\circ} 6,392-401$.

ETTINGTON, D.R.; 1998; Successful career plateauing; Journal of vocational behaviour, vol52, 72-88.

FELDMAN, D.C.; WEITZ, B.A.;1988; Career plateaus reconsidered; Journal of management, vol.14, $\mathrm{n}^{\circ} 1,69-80$.

FERENCE, T.P.; STONER, J.A.; WARREN, E.K.; 1977; Managing the career plateau; Academy of management review, october, 602-612.

LEMIRE, L. ; ROUILLARD, C. ; 2003 ; Le plafonnement de carrière : étude dans une municipalité au Québec ; Industrial Relations, vol. 58, n², 27 p.

SLOCUM, J.W.; CRON, W.L.; YOWS, L.; 1987; Career plateau: who's likely to plateau; business horizons; vol.30, ${ }^{\circ} 2,31-38$.

TREMBLAY, M. ; ROGER, A. ; 1995 ; Comment limiter les conséquences du plafonnement des carrières: une vérification empirique de trois pistes d'intervention auprès d'une population de cadres ; Carriérologie ; $9 \mathrm{p}$.

TREMBLAY, M.; ROGER, A.; 2004; Career plateauing reactions: the moderating role of job scope, role ambiguity and participation among Canadian managers; the international journal of human resource management, 15:6, september, 996-1017.

VEIGA, J.F.; 1981; Plateaued versus non-plateaued managers: career patterns, attitudes, and path potential; academy of management journal, vol.24, $n^{\circ} 3,566-578$.

ZAREMBA, D.K.; 1994; The managerial plateau: what helps in developing careers? ; The international journal of career management, vol.6, $n^{\circ} 2,5-11$. 